\title{
Duality transformations of Abelian and non-Abelian gauge fields*
}

\author{
Stanley Deser ${ }^{\dagger}$ \\ Physics Department, Harvard University, Cambridge, Massachusetts 02138 \\ Claudio Teitelboim \\ Joseph Henry Laboratories, Princeton University, Princeton, New Jersey 08540
}

(Received 10 December 1975)

\begin{abstract}
Duality transformations, i.e., rotations of electric and magnetic fields into each other, are implementable by a time-local generator for source-free Maxwell theory in an arbitrary spacetime metric. The Maxwell action $\int\left(E^{2}-B^{2}\right)$ as well as the stress tensor components, e.g., $E^{2}+B^{2}$, are duality invariant and the generator is conserved. In the formally analogous Yang-Mills case, duality transformations cannot even be consistently implemented.
\end{abstract}

\section{INTRODUCTION}

The source-free Maxwell equations, expressed in the symmetrical form

$$
\begin{gathered}
\partial_{\mu} F^{\mu \nu}=0, \quad \partial_{\mu}{ }^{\star} F^{\mu \nu}=0 ; \\
\star_{F^{\mu \nu}}=\frac{1}{2} \epsilon^{\mu \nu}{ }^{\alpha \beta} F_{\alpha \beta},
\end{gathered}
$$

are manifestly invariant under an arbitrary local linear transformation among the components of $F^{\mu \nu}$ and its dual $\star^{\mu \nu}$, or, equivalently, of $E^{i}$ and $B^{i}$. In particular, duality rotations, ${ }^{1}$ whose infinitesimal expression is $\delta \overrightarrow{\mathrm{E}}(x)=\beta \overrightarrow{\mathrm{B}}(x)$, $\delta \overrightarrow{\mathrm{B}}(x)=-\beta \overrightarrow{\mathrm{E}}(x)$, also leave the stress tensor $T^{\mu \nu}(F)$ invariant since its components are rotationally invariant binomials in $\overrightarrow{\mathrm{E}}$ and $\overrightarrow{\mathrm{B}}$. Paradoxically, the Maxwell action

$$
\begin{aligned}
S & =-\frac{1}{4} \int d^{4} x F_{\mu \nu} F^{\mu \nu} \\
& =\frac{1}{2} \int d^{4} x\left(E^{2}-B^{2}\right)
\end{aligned}
$$

would seem to be only invariant under hyperbolic rotations. Nevertheless, $S$ is easily seen to be invariant under infinitesimal rotations, at least, by recalling that in (1.2) $F_{\mu \nu}$ is only shorthand for $\partial_{\mu} A_{\nu}-\partial_{\nu} A_{\mu}$, for then

$$
\begin{aligned}
\delta_{\beta} S & =-\frac{1}{2} \int d^{4} x F^{\mu \nu} \delta F_{\mu \nu} \\
& =-\frac{1}{2} \beta \int d^{4} x F^{\mu \nu} F_{\mu \nu} \\
& =-\beta \int d^{4} x \partial_{\mu}\left(\epsilon^{\mu \nu \alpha \beta} A_{\nu} \partial_{\alpha} A_{\beta}\right)=0 .
\end{aligned}
$$

For finite rotations, however, the action would seem to change by a scale factor:

$$
\int\left(E^{\prime 2}-B^{\prime 2}\right)=\left(\cos ^{2} \beta-\sin ^{2} \beta\right) \int\left(E^{2}-B^{2}\right),
$$

which would paradoxically lead to a rescaled stress tensor, although the Euler-Lagrange equations would of course be unchanged.

The seemingly contradictory situation discussed above, that the field equations and stress tensor share a symmetry different from that of the action, is resolved by the remark that a formal transformation is only meaningful for a dynamical system if it can be implemented at the level of the basic field variables $\phi^{A}$ by a consistent set of variations $\delta \phi^{A}[\phi]$; in our case this would involve either the $A_{\mu}$ or the $A_{\mu}$ and their conjugates, depending on whether Lagrangian (second-order) or Hamiltonian (first-order) form is used. Any associated invariance will be meaningful if the corresponding variation of the action consists of a boundary term involving time derivatives of a finite order. This time locality requirement ensures that the boundary terms can have zero variation without the requirement that the field variations vanish for all times. Space nonlocality is permitted in the transformations provided only that the variations have sufficiently rapid falloff at spatial infinity.

We shall show that duality transformations in Maxwell theory can be implemented in terms of time-local variations in either Lagrangian ${ }^{2}$ or Hamiltonian formulation. The corresponding conserved generator is a simple gauge-invariant time-local functional of $\overrightarrow{\mathrm{E}}$ and $\overrightarrow{\mathrm{B}}$. Furthermore, the hyperbolic rotation which would be expected to keep the difference $\left(E^{2}-B^{2}\right)$ invariant cannot even be implemented in Lagrangian form and does not leave $\left(E^{2}-B^{2}\right)$ invariant in Hamiltonian form.

Non-Abelian gauge fields would seem to be quite analogous to the Maxwell case at first sight. There is a symmetric set of field equations

$$
\begin{gathered}
D_{\mu} \underline{F}^{\mu \nu}=0, D_{\mu}^{\star} \underline{F}^{\mu \nu}=0 ; \\
{ }^{\star} \underline{F}^{\mu \nu}=\frac{1}{2} \epsilon^{\mu \nu \alpha \beta} \underline{F}_{\alpha \beta}
\end{gathered}
$$

in terms of the covariant derivative $D_{\mu}=\partial_{\mu}+\underline{A}_{\mu} \times$ 
in the SU(2) case we will discuss for simplicity. (The underlines denote isovectors.) The stress tensor is precisely a sum of Maxwell-type terms; hence it is invariant under local $\left(\underline{F}_{\mu \nu}, \star^{\star} \underline{F}_{\mu \nu}\right)$ rotations. The action $S=-\frac{1}{4} \int \underline{F}_{\mu \nu} \cdot \bar{F}^{\mu \nu}$ is again seemingly invariant under infinitesimal linear transformations, since the integrand of $\delta_{\beta} S \sim \int_{F_{\mu \nu}}{ }^{\star} \underline{F}^{\mu \nu}$ is again a total divergence when the explicit from $\underline{F}_{\mu \nu}=\partial_{\mu} \underline{A}_{\nu}-\partial_{\nu} \underline{A}_{\mu}+\underline{A}_{\mu} \times \underline{A}_{\nu}$ is inserted. $^{3}$ However, we shall see that there exists no transformation of the basic fields which reduces to a duality rotation when the equations of motion hold and which leaves the action invariant. This conclusion will be reached in two different ways: (a) indirectly, by showing that there is no transformation of the $A_{\mu}$ which causes $F^{\mu \nu}$ and ${ }^{\star} F^{\mu \nu}$ to undergo a duality rotation while leaving (1.4) invariant and (b) by direct work on the action in the Hamiltonian formalism. In the latter approach, we can in fact separate the conclusion into two complementary statements: (i) There is no consistent way of reproducing $\left(F,{ }^{\star} F\right)$ rotations through basic field variations. (ii) There is no invariance of the action corresponding to the "nearest possible analog" of the rotations. In one view, the discrepancy between Abelian and non-Abelian systems can be traced to the fact that in the latter the longitudinal components of electric and magnetic fields are nonzero but rather complicated functions of the dynamical variables. Formally the problem lies in the dependence of $D_{\mu}$ on $A_{\mu}$ in the field equations (1.4), which must be taken into account when varying them.

Although we shall work mostly in a flat spacetime we will show explicitly that our conclusions carry over unchanged to an arbitrarily curved manifold.

\section{MAXWELL THEORY}

Hamiltonian form

The Maxwell action in first-order form can be considered at several successive steps of resolution:

$$
\begin{aligned}
S & =-\int d^{4} x\left[\overrightarrow{\mathrm{E}} \cdot \dot{\overrightarrow{\mathrm{A}}}+\frac{1}{2}\left(E^{2}+2 \overrightarrow{\mathrm{B}} \cdot \nabla \times \overrightarrow{\mathrm{A}}-B^{2}\right)+A_{0} \nabla \cdot \overrightarrow{\mathrm{E}}\right] \\
& =-\int d^{4} x\left\{\overrightarrow{\mathrm{E}} \cdot \dot{\overrightarrow{\mathrm{A}}}+\frac{1}{2}\left[E^{2}+(\nabla \times \overrightarrow{\mathrm{A}})^{2}\right]+A_{0} \nabla \cdot \overrightarrow{\mathrm{E}}\right\} \\
& =-\int d^{4} x\left\{\overrightarrow{\mathrm{E}}^{T} \cdot \dot{\overrightarrow{\mathrm{A}}}^{T}+\frac{1}{2}\left[\left(\overrightarrow{\mathrm{E}}^{T}\right)^{2}+\left(\nabla \times \overrightarrow{\mathrm{A}}^{T}\right)^{2}\right]\right\} \cdot(2.1 \mathrm{c})
\end{aligned}
$$

In (2.1a) all ten $E^{i}, B^{i}, A_{\mu}$ are independent; in (2.1b) the algebraic constraint $\vec{B}=\nabla \times \vec{A}$ has been eliminated; the Gauss constraint $\nabla \cdot \overrightarrow{\mathrm{E}}=0$ has been solved for in the final version (2.1c), where only the two pairs of gauge-invariant transverse vari- ables $\left(\overrightarrow{\mathrm{E}}^{T}, \overrightarrow{\mathrm{A}}^{T}\right)$ survive. For simplicity we work in the reduced form (2.1c), although we could have kept the additional gauge content $\left(A^{L}, A_{0}\right)$ of $(2.1 \mathrm{~b})$ as well as the $a$ priori independence of $\vec{B}$ and $\nabla \times \vec{A}$ of (2.1a).

We then define the finite rotation

$$
\begin{aligned}
& \overrightarrow{\mathrm{E}}^{\prime}=\cos \beta \overrightarrow{\mathrm{E}}+\sin \beta \nabla \times \overrightarrow{\mathrm{A}}, \\
& \nabla \times \overrightarrow{\mathrm{A}}^{\prime}=\cos \beta \nabla \times \overrightarrow{\mathrm{A}}-\sin \beta \overrightarrow{\mathrm{E}}
\end{aligned}
$$

in the space of transverse (divergence-free) vector fields, so that the vanishing of the logitudinal components of $(\overrightarrow{\mathrm{E}}, \overrightarrow{\mathrm{B}})$ is automatically incorporated in (2.2). The variation of $\vec{A}^{T}$ is uniquely obtained from that of $\nabla \times \overrightarrow{\mathrm{A}}^{T}$ by "inverting" with $-\nabla^{-2} \nabla \times$; infinitesimally we have

$$
\delta \overrightarrow{\mathrm{E}}=\beta \nabla \times \overrightarrow{\mathrm{A}}, \quad \delta \overrightarrow{\mathrm{A}}=\beta \nabla^{-2} \nabla \times \overrightarrow{\mathrm{E}} .
$$

The effect of the transformation (2.3) is to alter the Lagrangian by a total time derivative, i.e.,

$$
\delta_{\beta} S=-\left.\frac{1}{2} \beta \int d^{3} x\left(\overrightarrow{\mathrm{A}} \cdot \nabla \times \overrightarrow{\mathrm{A}}+\overrightarrow{\mathrm{E}} \cdot \nabla^{-2} \nabla \times \overrightarrow{\mathrm{E}}\right)\right|_{t_{1}} ^{t_{2}},
$$

so that (2.3) is indeed a symmetry transformation of the action (2.1c). The corresponding conserved generator is easily obtained: When the equations of motion hold, the change in the action is given by

$$
\delta S=\left.\int d^{3} x(-\overrightarrow{\mathrm{E}} \cdot \delta \overrightarrow{\mathrm{A}})\right|_{t_{1}} ^{t_{2}}=-\beta \int d^{3} x \overrightarrow{\mathrm{E}} \cdot \nabla^{-2} \nabla \times\left.\overrightarrow{\mathrm{E}}\right|_{t_{1}} ^{t_{2}} .
$$

Equating (2.4) and (2.5) we find that

$$
\begin{aligned}
G & =\frac{1}{2} \int d^{3} x\left(-\overrightarrow{\mathrm{A}} \cdot \nabla \times \overrightarrow{\mathrm{A}}+\overrightarrow{\mathrm{E}} \cdot \nabla^{-2} \nabla \times \overrightarrow{\mathrm{E}}\right) \\
& =\frac{1}{2} \int d^{3} x\left(\overrightarrow{\mathrm{B}} \cdot \nabla^{-2} \nabla \times \overrightarrow{\mathrm{B}}+\overrightarrow{\mathrm{E}} \cdot \nabla^{-2} \nabla \times \overrightarrow{\mathrm{E}}\right)
\end{aligned}
$$

is a constant of the motion. ${ }^{4}$ This is obvious in the second form since $\overrightarrow{\mathrm{B}}=-\nabla \times \overrightarrow{\mathrm{E}}, \dot{\overrightarrow{\mathrm{E}}}=+\nabla \times \overrightarrow{\mathrm{B}}$. It is also easy to check that $G$ generates the rotation (2.3), in the sense that the changes in $E$ and $A$ are given by the Poisson brackets of those quantities with $\beta G$.

Consider now the effect of a finite hyperbolic rotation in this formalism, namely

$$
\begin{aligned}
& \overrightarrow{\mathrm{E}}^{\prime}=\cosh \beta \overrightarrow{\mathrm{E}}+\sinh \beta \nabla \times \overrightarrow{\mathrm{A}}, \\
& \nabla \times \overrightarrow{\mathrm{A}}^{\prime}=\cosh \beta \nabla \times \overrightarrow{\mathrm{A}}+\sinh \beta \overrightarrow{\mathrm{E}} .
\end{aligned}
$$

It is easy to see that the $\int \overrightarrow{\mathrm{E}}^{T} \cdot \dot{\overrightarrow{\mathrm{A}}}^{T}$ part of the action is invariant, but the Hamiltonian is manifestly noninvariant, so the action when actually expressed as a functional of the basic variables is not invariant under hyperbolic rotations (but only 
is under ordinary rotations), despite its $\left(E^{2}-B^{2}\right)$ form.

Finally we show that our procedure carries over to the case when the spacetime is endowed with an arbitrary nonflat metric. Using the threedimensional notation $N=\left(-g^{00}\right)^{-1 / 2},{ }^{3} g=\operatorname{det}|| g_{i j}||$, $N^{i}={ }^{3} g^{i j} g_{0 j}$, where ${ }^{3} g^{i j}$ is the inverse of $g_{i j}$, together with the choice of Maxwell variables $\mathcal{E}^{i}=\sqrt{-g} F^{0 i}$, the generalization of $(2.1 c)$ reads $^{5}$

$$
\begin{gathered}
S=-\int d^{4} x\left[\mathcal{E}^{i} \dot{A}_{i}+\frac{1}{2} N\left({ }^{3} g\right)^{-1 / 2} g_{i j}\left(\mathcal{E}^{i} \mathcal{E}^{j}+\boldsymbol{B}^{i} \mathbb{B}^{j}\right)\right. \\
\left.-\epsilon_{i j k} N^{i} \mathcal{E}^{j} \mathbb{B}^{k}\right],
\end{gathered}
$$

where $\mathbb{B}^{i}=\epsilon^{i j k} \partial_{j} A_{k}$ is the magnetic field (a three density). The field $\Theta^{i}$ is clearly divergenceless, as is $\mathcal{E}^{i}$ which obeys the Gauss constraint $\mathcal{E}^{i}, i=0$ (ordinary and covariant divergences coincide for contravariant densities). Under the rotation $\delta \mathcal{E}^{i}=\beta \mathbb{B}^{i}, \delta \mathbb{B}^{i}=-\beta \mathcal{E}^{i}$, the last two terms in the integrand of (2.8) are manifestly invariant. To check the invariance of the $\mathcal{E}^{i} \dot{A}_{i}$ part we need not even explicitly obtain $\delta A_{i}^{T}$ (with $A_{i}=A_{i}^{T}+\partial_{i} \phi$, it is clear that only $A_{i}^{T}$ enters in either $B^{i}$ or the kinetic term since $\left.\mathcal{E}^{i}{ }_{i}=0\right)$. Using the general form $\mathcal{E}^{i}=\epsilon^{i j k} \partial_{j} Z_{k}$ for a divergenceless vector one obtains (dropping two-dimensional surface integrals at infinity)

$$
\delta_{\beta} S=-\left.\frac{1}{2} \beta \int d^{3} x \epsilon^{i j k}\left(A_{i} \partial_{j} A_{k}-Z_{i} \partial_{j} Z_{k}\right)\right|_{t_{1}} ^{t_{2}},
$$

so the action is indeed invariant. Expression (2.9) reduces to the more explicit form (2.4) when the space is flat.

\section{Lagrangian form}

We now consider how duality is expressed at the Lagrangian level. The action is

$$
\begin{aligned}
S & =\frac{1}{2} \int d^{4} x\left[\left(\dot{\overrightarrow{\mathrm{A}}}-\nabla A_{0}\right)^{2}-(\nabla \times \overrightarrow{\mathrm{A}})^{2}\right] \\
& \left.=\frac{1}{2} \int d^{4} x\left[\dot{\overrightarrow{\mathrm{A}}}^{T}\right)^{2}-\left(\nabla \times \overrightarrow{\mathrm{A}}^{T}\right)^{2}+\left(\dot{\overrightarrow{\mathrm{A}}}^{L}-\nabla A_{0}\right)^{2}\right],
\end{aligned}
$$

where we have separated transverse and longitudinal parts in the second form. It is clear from (2.10) that the transverse variables are decoupled from the longitudinal and gauge contributions. (Extremization of the action with respect to $A_{0}$ gives merely $\dot{\overrightarrow{\mathrm{A}}}^{L}=\nabla A_{0}$, which identifies $\overrightarrow{\mathrm{A}}^{L}$ as a gauge variable.) We can then drop the third term in (2.10) and work merely with

$$
S=\frac{1}{2} \int d^{4} x\left[\left(\dot{\overline{\mathrm{A}}}^{T}\right)^{2}-\left(\nabla \times \overrightarrow{\mathrm{A}}^{T}\right)^{2}\right] .
$$

The electric and magnetic fields are then given by $\overrightarrow{\mathrm{E}}=\dot{\overrightarrow{\mathrm{A}}}^{T}, \overrightarrow{\mathrm{B}}=\nabla \times \overrightarrow{\mathrm{A}}^{T}$. The infinitesimal duality rotation is written as

$$
\delta \overrightarrow{\mathrm{A}}^{T}=-\beta \nabla^{-2} \nabla \times \dot{\overrightarrow{\mathrm{A}}}^{T}
$$

and, of course, $\delta \dot{\vec{A}}^{T}=\left(\delta \overrightarrow{\mathrm{A}}^{T}\right)^{\dot{ }}$. It should be noted that (2.12) reproduces a duality rotation only on the mass shell; in fact we have $\delta \overrightarrow{\mathrm{B}}=\beta \overrightarrow{\mathrm{E}}$ all right, but the change in $\overrightarrow{\mathrm{E}}$ is given by $\delta \overrightarrow{\mathrm{E}} \equiv \delta \overrightarrow{\mathrm{A}}^{T}$ $\overline{\dot{A}}-\beta \nabla^{-2} \nabla \times \ddot{\bar{A}}^{T}$, which is equal to $-\beta \overrightarrow{\mathrm{B}}$ only if $\ddot{\overrightarrow{\mathrm{A}}}{ }^{T}=\nabla^{2} \overrightarrow{\mathrm{A}}^{T}$.

The change (2.12) alters the action by a boundary term,

$$
\delta_{\beta} S=-\left.\frac{1}{2} \beta \int d^{3} x\left(\overrightarrow{\mathrm{A}}^{T} \cdot \nabla \times \overrightarrow{\mathrm{A}}^{T}+\dot{\overrightarrow{\mathrm{A}}}^{T} \cdot \nabla^{-2} \nabla \times \dot{\overrightarrow{\mathrm{A}}}^{T}\right)\right|_{t_{1}} ^{t_{2}},
$$

and is therefore a symmetry transformation. The same conserved quantity (2.6) is associated with this invariance: First we note that on the mass shell the generic change in the action is given by

$$
\begin{aligned}
\delta S & =\left.\int d^{3} x \frac{\delta L}{\delta \dot{\overrightarrow{\mathrm{A}}}^{T}} \cdot \delta \overrightarrow{\mathrm{A}}^{T}\right|_{t_{1}} ^{t_{2}} \\
& =-\beta \int d^{3} x \dot{\overrightarrow{\mathrm{A}}}^{T} \cdot \nabla^{-2} \nabla \times\left.\dot{\overrightarrow{\mathrm{A}}}^{t}\right|_{t_{1}} ^{t_{2}} .
\end{aligned}
$$

Next we equate $(2.13)$ and $(2.14)$ to establish that $\frac{1}{2} \int d^{3} x\left(\dot{\mathrm{A}}^{T} \cdot \nabla^{-2} \nabla \times \dot{\mathrm{A}}^{T}-\overrightarrow{\mathrm{A}}^{T} \cdot \nabla \times \overrightarrow{\mathrm{A}}^{T}\right)$ is conserved, but this is precisely the generator (2.6).

Finally we note once again that hyperbolic rotations do not work. Indeed, once we start with (2.12), as we must in order to get $\delta \overrightarrow{\mathrm{B}}=\beta \overrightarrow{\mathrm{E}}$, the transformation is completely determined and it leads to $\delta \overrightarrow{\mathrm{E}}=-\beta \overrightarrow{\mathrm{E}}$, i.e., a circular rotation.

\section{YANG-MILLS FIELD}

The nonlinearity of non-Abelian theories greatly complicates the investigation of how duality might be implemented in that context. Fortunately we shall be able to show by general arguments that no transformation of the variables exists which (a) leaves the action invariant and (b) reduces to a duality rotation "on shell." We will then see concretely in Hamiltonian form why duality (let alone invariance) has no analog here.

The general proof is based on the elementary observation that invariance of the action implies invariance of the field equations, irrespective of formalism (Lagrangian or Hamiltonian), solution of constraints, or choice of gauge. Therefore, we consider the transformation of the Yang-Mills equations

$$
\partial_{\mu} \underline{F}^{\mu \nu}+\underline{A}_{\mu} \times \underline{F}^{\mu \nu}=0
$$

and of the identities 


$$
\partial_{\mu} \underline{F}^{\mu \nu}+\underline{A}_{\mu} \times{ }^{\star} \underline{F}^{\mu \nu}=0
$$

satisfied by $\star_{F}^{\mu \nu}=\frac{1}{2} \epsilon^{\mu \nu \rho \sigma} \underline{F}_{\rho \sigma}$, when $\underline{F}_{\mu \nu}$ has the form

$$
\underline{F}_{\mu \nu}=\partial_{\mu} \underline{A}_{\nu}-\partial_{\mu} \underline{A}_{\nu}+\underline{A}_{\mu} \times \underline{A}_{\nu} .
$$

We demand that there exists a set of variations $\delta A_{\mu}$, which will give on the mass shell

$$
\delta \underline{F}^{\mu \nu}=\beta^{\star} \underline{F}^{\mu \nu}, \quad \delta^{\star} \underline{F}^{\mu \nu}=-\beta \underline{F}^{\mu \nu} .
$$

We then find

$$
\begin{aligned}
& \left(\partial_{\mu}+\underline{A}_{\mu} \times\right) \delta \underline{F}^{\mu \nu}+\delta \underline{A}_{\mu} \times \underline{F}^{\mu \nu}=0, \\
& \left(\partial_{\mu}+\underline{A}_{\mu} \times\right) \delta^{\star} \underline{F}^{\mu \nu}+\delta \underline{A}_{\mu} \times \underline{F}^{\mu \nu}=0,
\end{aligned}
$$

which implies by (3.3) and (3.1) that

$$
\begin{aligned}
& \delta \underline{A}_{\mu} \times \underline{F}^{\mu \nu}=0, \\
& \delta \underline{A}_{\mu} \times{ }^{\star} \underline{F}^{\mu \nu}=0 .
\end{aligned}
$$

Relations (3.5) constitute a local system of homogeneous linear algebraic equations. To prove that it admits no nontrivial solutions for $\delta \underline{A}_{\mu}$ in the generic case it suffices to consider (3.5a), which reads

$$
M_{(a \nu)(b \mu)} \delta A^{(b \mu)}=0, \quad M_{(a \nu)(b \mu)}=\epsilon_{a b c} F_{c}^{\mu \nu} .
$$

The indices in the symmetic matrix $M$ run over $4 \times 3=12$ values in the $S U(2)$ case, and nontrivial solutions of (3.6) will exist if and only if $M$ has no inverse, i.e., if $\operatorname{det} M=0$. Now the block form of $M$ is

$$
M=\left[\begin{array}{ccc}
0 & F_{3} & -F_{2} \\
-F_{3} & 0 & F_{1} \\
F_{2} & -F_{1} & 0
\end{array}\right],
$$

where each block has an SU(2) label and elements within each block are labeled by $(\mu \nu)$. The nonvanishing of $\operatorname{det} M$ in four dimensions (it does vanish in two) can be checked explicitly using standard theorems on determinants ${ }^{6}$ or by the following argument. The inverse of $M$ is given by

$$
M^{-1}=\left[\begin{array}{ccc}
D_{1}^{-1} & D_{1}^{-1} F_{2} F_{1}^{-1} & D_{1}^{-1} F_{3} F_{1}^{-1} \\
D_{2}^{-1} F_{1} F_{2}^{-1} & D_{2}^{-1} & D_{2}^{-1} F_{3} F_{2}^{-1} \\
D_{3} F_{1} F_{3}^{-1} & D_{3}^{-1} F_{2} F_{3}^{-1} & D_{3}^{-1}
\end{array}\right],
$$

with

$$
D_{a}=\epsilon_{b a c} F_{b} F_{a}^{-1} F_{c} \quad(\text { no sum on } a),
$$

where $F_{a}{ }^{-1}$ is the inverse of $F_{a}^{\mu \nu}$ for fixed $a$ which exists in general since $\operatorname{det} F_{a}=\left(\overrightarrow{\mathrm{E}}_{a} \cdot \overrightarrow{\mathrm{B}}_{a}\right)^{2} \neq 0$. Now the $D$ 's obey the identities

$$
F_{a}^{-1} D_{b} F_{a}^{-1}=F_{b}^{-1} D_{a} F_{b}^{-1} \text { (no sum over } a \text { or } b \text { ) }
$$

which imply that the existence of $M^{-1}$ is equivalent to that of any $D_{a}^{-1}$, say $D_{1}^{-1}$. This is indeed the generic case and many solutions with nonsingular $D_{1}$ can be given. Incidentally, in two spacetime dimensions the $D_{a}$ are identically zero and consequently $M^{-1}$ does not exist in that case.

We have used the term "generic case" in the above as meaning arbitrary $F^{\mu \nu}$ rather than $A^{\mu}$. This practice is justified by $\bar{t}^{-}$purely local ${ }^{-}$ nature of the argument, which depends only on the value of $F^{\mu \nu}$ at a fixed point, $x_{0}$ say, rather than on the differential restrictions implied by its form [(3.2)] or the equation it obeys [(3.1a)] [in fact, given $F_{\mu \nu}\left(x_{0}\right)$ there is always a potential $A_{\mu}(x)$ which solves (3.1a) and (3.2) at $x_{0}$, namely $\left.\bar{A}_{\mu}(x)=\frac{1}{2} \underline{F}_{\nu \mu}\left(x_{0}\right)\left(x-x_{0}\right)^{\nu}+O\left(\left(x-x_{0}\right)^{3}\right)\right]$. Note, finally, that we have established the nonexistence of any duality rotations, including time-nonlocal ones, leaving the field equations invariant.

\section{An explicit calculation}

A concrete way of exhibiting the inconsistency of the duality concept in the non-Abelian case is to show that when the variations of the unconstrained variables in Hamiltonian form represent a rotation of the corresponding $(\vec{E}, \vec{B})$ components, the variations of the remaining ( $\overline{d e p e n d e n t) ~ c o m-~}$ ponents are not rotations and furthermore the action is not invariant. We proceed for explicitness in the axial gauge. There is no loss of generality in working in a specific gauge, since all field strengths transform in the same way under gauge changes.

The Yang-Mills action corresponding to the form $(2.1 \mathrm{c})$ is

$$
S=-\int d^{4} x\left[\underline{\overrightarrow{\mathrm{E}}} \cdot \underline{\dot{\mathrm{A}}}+\frac{1}{2}\left(\underline{\mathrm{E}}^{2}+\underline{\overrightarrow{\mathrm{B}}}^{2}\right)\right],
$$

where the covariantly divergenceless magnetic strength is given by $\underline{B}^{i}=(\nabla \times \overrightarrow{\mathrm{A}})^{i}+\frac{1}{2} \epsilon^{i j k} \underline{A}_{j} \times \underline{A}_{k}$. In the axial gauge one sets $A_{3}=0$ and solves the Gauss constraint $\nabla \cdot \underline{\vec{E}}+\underline{\vec{A}} \times \overrightarrow{\mathrm{E}}=\overline{0}$ as

$$
\underline{E}_{3}=-\int^{z} d \bar{z}\left(\underline{E}_{r, r}+\underline{A}_{r} \times \underline{E}_{r}\right), \quad r=1,2 .
$$

The action (3.11) depends then only on the unconstrained variables $\left(\underline{A}_{r}, \underline{E}_{r}\right)$ according to

$$
\begin{aligned}
-S=-S_{r}-S_{3}= & \int d^{4} x\left[\underline{E}_{r} \cdot \underline{\dot{A}}_{r}+\frac{1}{2}\left(\underline{E}_{r}{ }^{2}+\underline{B}_{r}{ }^{2}\right)\right] \\
& +\frac{1}{2} \int d^{4} x\left(\underline{E}_{3}{ }^{2}+\underline{B}_{3}{ }^{2}\right),
\end{aligned}
$$

where $\underline{E}_{3}$ is given by (3.12) and

$$
\underline{B}_{1}=-\partial_{3} \underline{A}_{2}, \quad \underline{B}_{2}=\partial_{3} \underline{A}_{1}, \quad \underline{B}_{3}=\partial_{1} \underline{A}_{2}-\partial_{2} \underline{A}_{1}+\underline{A}_{1} \times \underline{A}_{2} .
$$


Clearly the $r=1,2$ sector of (3.13) is of the Abelian Maxwell form and we must take

$$
\begin{aligned}
& \delta \underline{E}_{r}=\beta \underline{B}_{r}=-\beta \epsilon_{r s} \partial_{3} \underline{A}_{s}, \\
& \delta \underline{B}_{r}=-\beta \underline{E}_{r} \Longleftrightarrow \delta \underline{A}_{r}=-\beta \epsilon_{r s} \int^{z} d \bar{z} \underline{E}_{s} .
\end{aligned}
$$

The induced transformations of the independent variables $\underline{E}_{3}, \underline{B}_{3}$ are then

$$
\begin{aligned}
& \delta \underline{E}_{3}=\beta\left[\underline{B}_{3}-\left(\int^{z} d \bar{z} \underline{E}_{1}\right) \times\left(\int^{z} d \bar{z} \underline{E}_{2}\right)\right], \\
& \delta \underline{B}_{3}=\beta\left(-\underline{E}_{3}+\int^{z} d \bar{z} \underline{E}_{r} \times \underline{A}_{r}+\underline{A}_{r} \times \int^{z} d \bar{z} \underline{E}_{r}\right),
\end{aligned}
$$

which means that $\underline{E}_{3}, \underline{B}_{3}$ do not rotate into each other if the $r$ components do. Nor is the action left invariant, since the last part of (3.13) acquires a volume term:

$$
\begin{aligned}
\delta S_{3}=\beta \int d^{4} x[ & \left(\int^{z} d \bar{z}_{E_{1}} \times \int^{z} d \bar{z}_{E_{2}}\right) \cdot \underline{E}_{3} \\
& \left.-\left(\underline{A}_{r} \times \int^{z} d \bar{z} \underline{E}_{r}-\int^{z} d \bar{z} \underline{A}_{r} \times \underline{E}_{r}\right) \cdot \underline{B}_{3}\right] .
\end{aligned}
$$

Note that once the necessary choice (3.15) was made there was no freedom left to save that part of the transformation involving the constrained sector.

\section{SUMMARY}

We have seen that the concept of a duality rotation can be implemented in a satisfactory way in the source-free ${ }^{7}$ Maxwell theory in an arbitrary metric, and that the duality operation is a symmetry of the action with a corresponding generator. This justifies a recent application ${ }^{8}$ of the invariance to restrict possible counterterms in the quantized Einstein-Maxwell ${ }^{9}$ system to involve only the duality-invariant local functions of $F_{\mu \nu}$ in the relevant order, namely $T_{\mu \nu}$ and $\left(F^{\mu \nu} ; \nu\right)^{2}$.

In the Yang-Mills case we saw that no transformation $\delta \underline{A}_{\mu}$ exists which both induces the desired rotations and leaves the action invariant. This is in spite of the formal analogy to the Maxwell case and the explicit duality invariance ${ }^{10}$ of $T_{\mu \nu}^{\mathrm{YM}}$.

The methods and conclusions presented here for vector fields (spin one) can be carried over to general relativity, where the basic variables are tensor fields (spin two). There we find that the linearized theory is invariant under rotations of the unconstrained, transverse-traceless variables $b_{i j}^{\mathrm{TT}}, \pi^{i j \mathrm{TT}}$ (here $b_{i j}^{\mathrm{TT}}$ is an appropriate functional of the canonical coordinate $h_{i j}^{\mathrm{TT}}$ conjugate to $\pi^{i j \mathrm{TT}}$ ), but the invariance is no longer maintained in the full, self-interacting theory. Those results will be reported elsewhere.

Supplementary note. We understand that some of the duality problems of the Yang-Mills theory have been recently discussed by Gu Chao-hao and C. N. Yang. ${ }^{11}$

\section{ACKNOWLEDGMENTS}

S. D. thanks the Princeton University Physics Department for hospitality while part of this research was carried out. C. T. wishes to thank J. A. Wheeler for constant encouragement.
*Work supported in part by the National Science Foundation under Grants No. GP30799X and No. MPS 7417849A01.

$\dagger$ On leave from Brandeis University, Waltham, Mass. 02154.

${ }^{1}$ C. W. Misner and J. A. Wheeler, Ann. Phys. (N.Y.) 2 525 (1957)

${ }^{2}$ In this form the transformation reduces to a duality rotation "on the mass shell," i.e., when the Maxwell equations hold.

${ }^{3}$ The variation is given by

$$
\delta_{\beta} S=-\beta \int d^{4} x \partial_{\mu}\left[\epsilon^{\mu \nu \alpha \beta_{A}} \underline{\nu}_{\nu} \cdot\left(\partial_{\alpha} \underline{A}_{\beta}+\frac{1}{3} \underline{A}_{\alpha} \times A_{\beta}\right)\right] .
$$

${ }^{4}$ There is actually an infinity of different conserved quantities of the form (2.6), obtained by replacing $\nabla^{-2}$ by any $f\left(\nabla^{2}\right)$. The "local" constant with $f=1$ was discussed by D. Lipkin, J. Math. Phys. 5 , 696 (1974). Note also that by writing $\overrightarrow{\mathrm{E}}^{T}$ in potential form $\overrightarrow{\mathrm{E}}=\nabla \times \overrightarrow{\mathrm{Z}}$, we can cast $(2.6)$ in a local form,

$$
G=-\frac{1}{2} \int d^{3} x(\overrightarrow{\mathrm{A}} \cdot \nabla \times \overrightarrow{\mathrm{A}}+\overrightarrow{\mathrm{Z}} \cdot \nabla \times \overrightarrow{\mathrm{Z}}) .
$$

${ }^{5}$ R. Arnowitt, S. Deser, and C. W. Misner, Phys. Rev. 120,313 (1960).

${ }^{6} \mathrm{~T} . \mathrm{T}$. Wu and C. N. Yang (private communication) have carried out the explicit calculation and obtained

$\operatorname{det} M=\left[3^{-1} 2^{-5}\left(\underline{F}_{\mu \nu} \cdot \underline{F}_{\alpha \beta} \times \underline{F}_{\rho \sigma}\right)\left({ }^{\star} \underline{F}^{\mu \nu}{ }^{\star} \underline{F}^{\alpha \beta} \times^{\star} \underline{F}^{\rho \sigma}\right)\right]^{2}$.

${ }^{7}$ For a recent discussion of the Maxwell case with electric and magnetic sources see J. Schwinger, Phys. Rev. D 12, 3105 (1975).

${ }^{8}$ S. Deser, M. Grisaru, P. van Nieuwenhuizen, and C. C. Wu, Phys. Lett. 58B, 355 (1975).

${ }^{9}$ The same restriction can then be deduced to hold for the (non-duality-invariant) Yang-Mills case because a separate argument [S. Deser, P. van Nieuwenhuizen, and H. S. Tsao, Phys. Rev. D 10, 3337 (1974)] shows that the two systems will necessarily have counterterms of the same form, whatever these may be.

${ }^{10}$ The natural question whether there exists a non-Abe- 
lian analog to the "already unified theory" of Ref. 1 faces the major difficulty that the stress tensor is no longer idempotent. However, should there be some other way of obtaining $\underline{F}^{\mu \nu}$ from the geometry up to an admixture of ${ }^{\star} F^{\mu \nu}$, as is the case in Einstein-Maxwell theory, then the lack of duality invariance here would eliminate this ambiguity.

${ }^{11} \mathrm{Gu}$ Chao-hao and C. N. Yang, Sci. Sin. 18, 483 (1975). 